\title{
Face-Dependent Bond Lengths in Molecular Chemisorption: The Formate Species on $\mathrm{Cu}(111)$ and $\mathrm{Cu}(110)$
}

\author{
D. Kreikemeyer-Lorenzo, ${ }^{1}$ W. Unterberger, ${ }^{1}$ D. A. Duncan, ${ }^{2}$ M. K. Bradley, ${ }^{2}$ T. J. Lerotholi, ${ }^{2, *}$ \\ J. Robinson, ${ }^{2}$ and D. P. Woodruff ${ }^{2, \dagger}$ \\ ${ }^{1}$ Department of Chemical Physics, Fritz-Haber-Institut der MPG, Faradayweg 4-6, 14195 Berlin, Germany \\ ${ }^{2}$ Physics Department, University of Warwick, Coventry, CV4 7AL, United Kingdom
}

(Received 28 April 2011; published 22 July 2011)

\begin{abstract}
Many previous structural studies of molecular adsorbates on metal surfaces indicate that the local coordination and bonding is closely similar to that in organometallic compounds, implying that the metallic substrate has no significant influence. Here we show that such an influence is detectable for one model system, namely, the formate species, HCOO, adsorbed on the atomically rough and smooth (110) and (111) surfaces of $\mathrm{Cu}$, leading to a statistically significant difference $(0.09 \pm 0.05 \AA)$ in the $\mathrm{Cu}-\mathrm{O}$ chemisorption bond length. The effect is reproduced in density functional theory calculations.
\end{abstract}

DOI: 10.1103/PhysRevLett.107.046102

PACS numbers: $68.43 . \mathrm{Fg}, 68.43 . \mathrm{Bc}$

Chemisorption of molecular species on metal surfaces has been found to follow closely the bonding behavior of these same species in organometallic compounds [1]. Perhaps the most obvious and widely studied example is the similarity between $\mathrm{CO}$ bonding to metal surfaces and that in metal carbonyl complexes. Not only does $\mathrm{CO}$ adopt, on different surfaces or at different coverages, mainly onefold and twofold, (but also threefold) coordination to the surface metal atoms in a similar fashion to that in carbonyl complexes, but the change in the C-metal chemisorption bond length with coordination, and thus with bond order, has also been shown to follow Pauling-like behavior [2]. The strength of this similarity in local metal-molecule bonding seems surprising, because the atoms at the surface of a metal solid are not isolated, but are bonded to many other metal atoms, albeit through delocalized metallic bonding. Apparently the local surface-atom/molecule bond is relatively uninfluenced by the presence of the underlying bulk metal, yet one might certainly expect this to have some influence on the chemisorption bond length and adsorption energy. Here we provide the first clear and quantitative evidence that this is, indeed, the case. To achieve this, we have studied a molecule/metal system in which the local bonding coordination of the adsorbate molecule is the same on two crystallographic orientations of the metal surface that differ substantially in their "atomic roughness." Moreover, by using a common experimental methodology (based on the well-established method of scanned-energy mode photoelectron diffraction $(\mathrm{PhD})$ [3]) we minimize the impact of technique-related systematic errors on measured differences in bond lengths. Specifically, we report the results for adsorption of the formate species, $\mathrm{HCOO}$, on the close-packed $\mathrm{Cu}(111)$ surface, and on the more open-packed, atomically corrugated, $\mathrm{Cu}(110)$ surface. The results confirm that the local molecular bonding geometry and coordination is identical on these two surfaces, but we find a small but significant chemisorption bond length difference on the two different crystal faces. We also show that this effect of the underlying solid is reproduced qualitatively in the results of density functional theory (DFT) calculations, which also show a significant difference in adsorption energy on the two surfaces.

Quantitative structure determinations have previously been conducted on $\mathrm{Cu}$ surfaces exposed to a range of carboxylic acids, including formic $(\mathrm{HCOOH})$ [4-8], acetic $\left(\mathrm{CH}_{3} \mathrm{COOH}\right)[9,10]$, and benzoic $\left(\mathrm{C}_{6} \mathrm{H}_{5} \mathrm{COOH}\right)$ [11] acid. Most studies have been on $\mathrm{Cu}(110)$, but a small number of investigations have been performed on $\mathrm{Cu}(100)$ and $\mathrm{Cu}(111)$. In all cases the chemisorbed species is found to be a deprotonated carboxylate, $\mathrm{RCOO}$, with the molecule bridging two nearest-neighbor $\mathrm{Cu}$ atoms in the surface such that each $\mathrm{O}$ atom is onefold coordinated to $\mathrm{a} \mathrm{Cu}$ atom. Indeed, this carboxylate/ $\mathrm{Cu}$ bonding coordination is also found to extend to the simple amino acids, glycine $\left(\mathrm{NH}_{2} \mathrm{CH}_{2} \mathrm{COOH}\right)$ [12] and alanine $\left(\mathrm{NH}_{2} \mathrm{CH}_{3} \mathrm{CHCOOH}\right)$ [13] on $\mathrm{Cu}(110)$ and $\mathrm{Cu}(100)$, although for these molecules additional molecule-substrate bonding arises through the amino $\mathrm{N}$ atom. For the simple carboxylates, the symmetric $\mathrm{Cu}-\mathrm{O}$ bonds are almost perpendicular to the surface with the molecule "standing up." In fact the first study of the $\mathrm{Cu}(100) /$ formate system led to the conclusion that the adsorption geometry was quite different, and was cited as an example of a system in which the surface bonding was quite different from that of the organometallic analogues [7]. However, subsequent studies, including reanalysis of the data [4], showed this conclusion to be incorrect, and confirmed the common local coordination geometry of all of these systems. The results of these previous structural studies using $\mathrm{PhD}$ (with a more approximate analysis methodology), SEXAFS (surface extended x-ray absorption fine structure), and NIXSW (normal incidence X-ray standing waves) are summarized in Table I. In all cases the $\mathrm{Cu}-\mathrm{O}$ bond length is found to be in the range 1.91-1.99 $\AA$, 
very similar to the values in $\mathrm{Cu}_{2}$ formate complexes in which formate species bridge the two $\mathrm{Cu}$ atoms (e.g., $1.98 \AA$ [14] and $1.96 \AA$ [15]). However, as several different experimental methods have been used in these surface studies, with different intrinsic precision and different potential sources of systematic errors, it is impossible to draw any reliable conclusions regarding any facedependence of these bond lengths. Indeed, based on an uncritical evaluation of the values in this table, one might infer that the $\mathrm{Cu}-\mathrm{O}$ bond length is shorter on $\mathrm{Cu}(111)$ than on $\mathrm{Cu}(110)$, whereas the opposite proves to be the case.

To obtain a more meaningful comparison that explores the influence of the different metal surface structures, we have used the $\mathrm{PhD}$ technique to determine the local structure of the formate species on both the most atomically corrugated and the most atomically-smooth low-index $\mathrm{Cu}$ surfaces, namely, (110) and (111). This technique exploits the interference of the directly emitted component of the photoelectron wave field from a core level of an adsorbate atom (O $1 s$ in the present case) with other components of the same wave field elastically scattered from the surrounding substrate atoms. Modulations in the photoemission signal as the photon energy, and thus photoelectron wavelength, change are dependent on the local scattering path lengths and thus the emitter-substrate geometry. The method has been shown to be highly effective in determining the structure of a large number of adsorbate/substrate systems [3], and the use of the same technique to study both surfaces minimizes the influence of any possible systematic errors on the relative values of the $\mathrm{Cu}-\mathrm{O}$ bond lengths. Structure determination is achieved through comparison of the experimental $\mathrm{PhD}$ modulation spectra with the results of multiple-scattering simulations, performed using computer codes developed by Fritzsche [16-18], for a range of iterated model structures until the best fit was

TABLE I. Summary of previous determinations of the $\mathrm{Cu}-\mathrm{O}$ bond length for carboxylate species on $\mathrm{Cu}$ surfaces.

\begin{tabular}{llll}
\hline \hline Substrate & Adsorbate & $\begin{array}{l}\text { Technique } \\
\text { [reference] }\end{array}$ & $\begin{array}{l}\text { Cu-O bond } \\
\text { length }(\AA)\end{array}$ \\
\hline $\mathrm{Cu}(110)$ & Formate & $\begin{array}{l}\text { SEXAFS [4] } \\
\text { (reanalysis of [5]) }\end{array}$ & $1.94 \pm 0.10$ \\
& Formate & PhD $^{\mathrm{a}}[6]$ & $1.98 \pm 0.04$ \\
& Acetate & PhD [9] & $1.91 \pm 0.04$ \\
& Benzoate & PhD [11] & $1.91 \pm 0.02$ \\
$\mathrm{Cu}(100)$ & Formate & SEXAFS [4] & $1.99 \pm 0.10$ \\
& & (reanalysis of [7]) & \\
& Formate & PhD [6] & $1.98 \pm 0.04$ \\
$\mathrm{Cu}(111)$ & Formate & NIXSW [8] & $1.92 \pm 0.04^{\mathrm{b}}$ \\
& Acetate & NIXSW [10] & $1.86 \pm 0.04^{\mathrm{b}}$ \\
\hline \hline
\end{tabular}

These studies used a simplified theoretical modeling that may involve some systematic error.

${ }^{b}$ NIXSW does not measure the bond length directly, so these values rely on certain assumptions about the surface relaxation. obtained as judged by an objective reliability factor or $R$ factor [3]. The final structural precision was defined by a variance as described elsewhere [19].

The experiments were conducted in an ultrahigh vacuum surface science end-station installed on the UE56/2-PGM2 beamline of BESSY II [20]. Sample characterization was achieved by low energy electron diffraction (LEED), and by soft-x-ray (synchrotron radiation) photoelectron spectroscopy (SXPS), using an Omicron EA-125HR electron analyzer mounted at $60^{\circ}$ to the incident $X$ radiation. Several cycles of $500 \mathrm{eV}$ Ar ion bombardment followed by brief annealing to $\sim 670^{\circ} \mathrm{C}$ led to clean well-ordered surfaces, as determined by SXPS and LEED. The surfaces were then exposed to $\sim 5 \times 10^{-6}$ mbar.s of formic acid at $\sim 150 \mathrm{~K}$, followed by brief annealing to $300 \mathrm{~K}$. O $1 s$ and $\mathrm{C}$ $1 s$ SXPS confirmed that the resulting surface species was formate. $\mathrm{O} 1 s \mathrm{PhD}$ data, recorded in several emission directions, were collected and processed following standard procedures described elsewhere [3].

Figure 1 shows schematic diagrams of the structures obtained for the two systems, while Fig. 2 shows a comparison of the experimental and simulated $\mathrm{PhD}$ modulation spectra for this best-fit structure. The results confirm the local adsorption site found in earlier studies. The important new result is that the $\mathrm{Cu}-\mathrm{O}$ bond lengths are found to be $1.90 \pm 0.03 \AA$ on $\mathrm{Cu}(110)$ and $1.99 \pm 0.04 \AA$ on $\mathrm{Cu}(111)$. This difference in bond lengths is statistically significant $(0.09 \pm 0.05 \AA)$, and indeed inspection of the PhD modulation spectra of Fig. 2 shows a clear difference in the peak positions and periodicity from the two surfaces in the nearneighbor backscattering direction $\left(0^{\circ}\right)$, a direct spectral signature of the difference in bond lengths.

Two related differences between $\mathrm{Cu}(111)$ and $\mathrm{Cu}(110)$ surfaces can be identified as probable sources of this chemisorption bond length difference. One is the Smoluchowski smoothing of the metal valence electron density at the surface of the atomically corrugated $\mathrm{Cu}(110)$ surface that leaves surface $\mathrm{Cu}$ atoms depleted of valence charge, relative to those on the atomically smooth (111) surface. The other is the lower coordination of the surface atoms, relative to those in the surrounding bulk metal, on $\mathrm{Cu}(110)$ (6), than on $\mathrm{Cu}(111)$ (9). Both effects will lead to a shorter chemisorption bond length on the (110) surface. One additional difference between the two

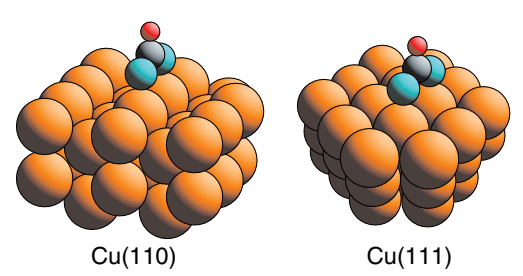

FIG. 1 (color online). Schematic diagrams of the local adsorption structure of the formate species on $\mathrm{Cu}(110)$ and $\mathrm{Cu}(111)$ as determined in this study. 


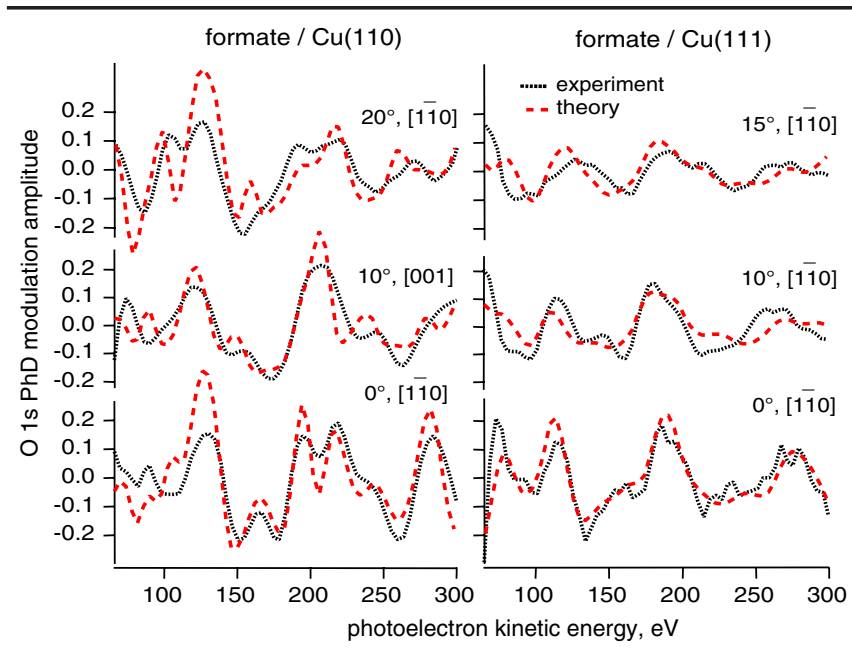

FIG. 2 (color online). Comparison of the experimental O $1 s$ $\mathrm{PhD}$ modulations spectra, recorded in several different emission directions (polar angle and azimuth) from the adsorbed formate species on $\mathrm{Cu}(110)$ and $\mathrm{Cu}(111)$, with the results of multiplescattering simulations for the best-fit structures.

surfaces which could influence the bond lengths is the significantly larger surface dipole moment of the (110) surface; this is, of course, also a consequence of the Smoluchowski smoothing at this more atomically corrugated surface.

To provide further insight into the experimental result we have performed DFT calculations using the CASTEP5.0 pseudopotential plane-wave [21] code, with 5-layer slabs to represent the $\mathrm{Cu}$ substrates, one formate species per $(3 \times 2)$ mesh, ultrasoft pseudopotentials, $6 \times 6$ [(110)] and $6 \times 8$ [(111)] Monkhorst-Pack $k$-point sampling, and 420 eV energy cutoff. Both RPBE (revised Perdew-BurkeEnzerhof) and $\mathrm{PBE}$ functionals yielded $\mathrm{Cu}-\mathrm{O}$ bond lengths that are $0.04 \AA$ longer on the (111) surface than on (110), qualitatively consistent with the experiment result. The absolute values of the bond lengths in DFT are larger than the experimental values (e.g., for (111) the RBPE and PBE values are $2.04 \AA$ and $2.01 \AA$, to be compared with the experimental value of $1.99 \AA$ ), but discrepancies between experiment and theory of up to $0.1 \AA$ are common in molecular adsorption systems. A comparison of calculations on $\mathrm{Cu}(110)$ for formate adsorbed on one side of a 5-layer slab, and on both sides of a 9-layer slab, respectively, showed no difference in bond lengths, demonstrating that the calculated bond lengths are not influenced by surface dipole-derived fields in the supercell. In order to check on any possible influence of van der Waals interactions, CASTEP DFT-D (Dispersion) calculations were also performed. These semiempirical methods are known to have limited applicability to adsorption on metal surfaces, but have nevertheless proved effective in some systems [22]. In the present case, the inclusion of the correction for dispersion forces led to no significant changes in the bond lengths. The DFT results thus confirm that the bond length difference can be attributed entirely to changes in the metallic and covalent bonding at the two surfaces. While the calculated Mulliken charges showed a significant $\left(0.24 e^{-}\right)$depletion of valence charge at the $\mathrm{Cu}$ atoms on the (110) surface relative to those on the (111) surface, as expected from the preceding arguments, the net charge transfer associated with the formate bonding was essentially identical. Moreover, no clear signature of the bonding difference was seen in the local charge distribution around the $\mathrm{Cu}-\mathrm{O}$ bond, nor in the local density of states in the molecular orbitals. Nevertheless, the results showed that an increase in adsorption energy on the (110) surface of $\sim 0.4 \mathrm{eV} /$ molecule, relative to that on the (111) surface, accompanies the shorter $\mathrm{Cu}-\mathrm{O}$ bond length.

In conclusion, our results demonstrate that even in a system in which most details of molecular bonding at metal surfaces follow closely the behavior of organometallic compounds, there are subtle manifestations of the fact that the metal atoms are part of a solid surface, leading to changes in bond length (and adsorption energy) at locally equivalent sites on different crystallographic faces of the same solid. The influence on the bond length is not large, nor would it be expected to be if the chemisorption coordination is the same, but the DFT calculations indicate that the influence on the adsorption energy $(\sim 0.4 \mathrm{eV})$ is substantial.

This work was funded in part by the Engineering and Physical Sciences Research Council (UK).

*Present address: School of Chemistry, University of Witwatersrand, PO Wits, Johannesburg, 2050, South Africa.

${ }^{\dagger}$ Corresponding author

d.p.woodruff@warwick.ac.uk

[1] M. R. Albert and J. T. Yates, Jr., The Surface Scientist's Guide to Organometallic Chemistry (Am. Chem. Soc., Washington, DC (USA), 1987).

[2] D. I. Sayago et al., Phys. Rev. Lett. 90, 116104 (2003).

[3] D. P. Woodruff, Surf. Sci. Rep. 62, 1 (2007).

[4] M. D. Crapper, C. E. Riley, and D. P. Woodruff, Surf. Sci. 184, 121 (1987).

[5] A. Puschmann, J. Haase, M. D. Crapper, C. E. Riley, and D. P. Woodruff, Phys. Rev. Lett. 54, 2250 (1985).

[6] D. P. Woodruff et al., Surf. Sci. 201, 228 (1988).

[7] J. Stöhr, D. A. Outka, R. J. Madix, and U. Döbler, Phys. Rev. Lett. 54, 1256 (1985).

[8] A. Sotiropoulos et al., Surf. Sci. 444, 52 (2000).

[9] K.-U. Weiss et al., Phys. Rev. Lett. 69, 3196 (1992).

[10] S. M. Johnston et al., Surf. Sci. 477, 163 (2001).

[11] M. Pascal et al., Surf. Sci. 492, 285 (2001).

[12] J.-H. Kang et al., J. Chem. Phys. 118, 6059 (2003).

[13] D. I. Sayago et al., Surf. Sci. 590, 76 (2005).

[14] D. M. L. Goodgame et al., J. Chem. Soc. D: Chem. Commun., 629 (1969).

[15] A. Golobic, M. Malekovic, and P. Šegedin, Acta Crystallogr. Sect. C 62, m102 (2006). 
[16] V. Fritzsche, J. Phys. Condens. Matter 2, 1413 (1990).

[17] V. Fritzsche, Surf. Sci. 265, 187 (1992).

[18] V. Fritzsche, Surf. Sci. 213, 648 (1989).

[19] N. A. Booth et al., Surf. Sci. 387, 152 (1997).
[20] K. J. S. Sawhney et al., Nucl. Instrum. Methods Phys. Res., Sect. A 390, 395 (1997).

[21] S. J. Clark et al., Z. Kristallogr. 220, 567 (2005).

[22] G. Mercurio et al., Phys. Rev. Lett. 104, 036102 (2010). 